\title{
Weyl-van der Waerden formalism for helicity amplitudes of massive particles
}

\author{
Stefan Dittmaier \\ Theory Division, CERN, CH-1211 Geneva 23, Switzerland \\ (Received 11 August 1998; published 10 December 1998)
}

\begin{abstract}
The Weyl-van der Waerden spinor technique for calculating helicity amplitudes of massive and massless particles is presented in a form that is particularly well suited to a direct implementation in computer algebra. Moreover, we explain how to exploit discrete symmetries and how to avoid unphysical poles in amplitudes in practice. The efficiency of the formalism is demonstrated by giving explicit compact results for the helicity amplitudes of the processes $\gamma \gamma \rightarrow f \bar{f}, f \bar{f} \rightarrow \gamma \gamma \gamma, \mu^{-} \mu^{+} \rightarrow f \bar{f} \gamma$. [S0556-2821(99)02101-3]

PACS number(s): 11.55.Bq, 11.80.Cr, 12.15.Ji
\end{abstract}

\section{INTRODUCTION}

The calculation of scattering amplitudes and cross sections in the lowest order of perturbation theory is one of the standard problems of elementary particle phenomenology in practice. Although this task is very simple for $1 \rightarrow 2$ particle decays and $2 \rightarrow 2$ particle reactions, the situation can become arbitrarily complicated for more particles in the final state, as the number $N_{D}$ of contributing Feynman diagrams rapidly increases. Squaring amplitudes and using completeness relations for the wave functions does not represent an appropriate approach if $N_{D}$ is large, because one would get $N_{D}^{2}$ contributions. A reasonable way to avoid an explosion of the algebraic work consists in using spinor formalisms for the evaluation of amplitudes for definite polarization configurations. The squaring of the amplitudes and the spin summations are then performed numerically. Of course, the number of different polarization configurations also grows for more and more external particles, but in practice this fact is much less problematic than squaring amplitudes. In particular, the number of independent matrix elements can be reduced by exploiting discrete symmetries. Although the commonly applied spinor formalisms rely on the four dimensionality of space-time, they are also useful for the calculation of higherorder corrections if the singularities in dimensional regularization are split off and controlled separately.

Various spinor techniques have been proposed and successfully applied in recent years. The first versions [1] proposed a clever choice of photon and gluon polarization vectors, which was dictated by the momenta of the attached fermions. This choice forces a lot of terms in the calculation to vanish, and relatively compact amplitudes result. Although the initial restriction to massless particles could be overcome [2], all those formalisms have in common that the usual Dirac algebra is still present. The final results for amplitudes are given in terms of standard products $\bar{u}_{1}\left(p_{1}\right) u_{2}\left(p_{2}\right)$ of Dirac spinors. This form is useful for numerical evaluations, but too involved for further analytical purposes.

It is more elegant and transparent to express all needed ingredients, i.e., momenta, polarization vectors, and Dirac spinors, in terms of a single mathematical object. This leads us to two-component Weyl-van der Waerden (WvdW) spinors [3], which form the fundamental representations of the Lorentz group, and the related spinor calculus (see also Ref. [4]). Since WvdW spinors are closely related to lightlike vectors, the application of the WvdW spinor calculus is extremely simple for massless particles. Such applications can be found, e.g., in Ref. [5]. The WvdW technique can also be used for massive particles if timelike momenta are decomposed into two lightlike ones. This procedure was applied in Refs. [6,7] for the calculation of bremsstrahlung corrections to production processes of massive weak gauge bosons, leading to rather compact results. However, the WvdW spinor formalism for massive particles is not yet well documented in the literature. In connection with the actual applications presented in Refs. [6,7], the formalism was only briefly sketched, as far as was relevant for the special cases under investigation. More complete presentations of the technique for massive particles are either not suited for direct practical use [8] or appeared in unpublished studies $[9,10]$.

In addition to the more technical motivation above for considering amplitudes with definite polarization configurations, one should add some remarks on the physical role of polarized massive particles. In contrast with massless particles, the spin orientations of massive particles do not transform covariantly under Lorentz transformations; in particular, the property of being a helicity eigenstate is frame dependent. Nevertheless, the polarization states of a massive particle also carry valuable physical information about the structure of its interaction. For instance, the longitudinal modes of the massive weak gauge bosons serve as a window into the scalar sector of the electroweak theory, and the reconstruction of the top-quark spin from its decay products reveals information about its static properties and form factors. For various aspects of physics with polarized massive particles, see Ref. [11] and references therein.

The aim of this paper is threefold. First, we give an introduction to the WvdW spinor technique for massive and massless particles. The presentation is deliberately held at a basic and detailed level, in order to facilitate the practical use of the method. In particular, we present all ingredients needed for an implementation in computer algebra. As wave functions for massive spin- $\frac{1}{2}$ and spin-1 particles, we take the helicity eigenstates formulated in Ref. [10], which appear to be very simple. The wave functions and the momenta of the corresponding massive particles are composed of the same set of auxiliary spinors, leading to simplifications in the calculation of amplitudes. Second, we show how to exploit the 
advantages of the formalism in practice. We explain the use of discrete symmetries, in order to reduce the algebraic work, and give a prescription for avoiding unphysical poles in amplitudes. In the presented examples, we treat the polarizations of massive fermions in a generic way; i.e., explicit results for definite helicity configurations follow from a few compact generic amplitudes upon setting auxiliary spinors to specific values. Third, we give explicit results for helicity amplitudes of phenomenologically relevant processes involving massive particles. We explicitly treat the reactions $\gamma \gamma$ $\rightarrow f \bar{f}, f \bar{f} \rightarrow \gamma \gamma \gamma, \mu^{-} \mu^{+} \rightarrow f \bar{f} \gamma$ in the framework of the electroweak standard model and show how these results can be carried over to other processes such as $\gamma \gamma \rightarrow f \bar{f} \gamma, e^{-} \gamma$ $\rightarrow e^{-} \gamma \gamma, e^{-} \gamma \rightarrow e^{-} e^{-} e^{+}$by using crossing relations.

The outline of the paper is as follows. In Sec. II we set our basic conventions for the WvdW spinor calculus and introduce helicity eigenstates for spin $-\frac{1}{2}$ and spin- 1 particles. The necessary ingredients for calculating helicity amplitudes are described in Sec. IV, specifically containing a prescription for translating Feynman rules into the WvdW formalism, a formulation of discrete symmetries for helicity amplitudes, and a prescription for avoiding unphysical poles in amplitudes. The physical applications are presented in Sec. $\mathrm{V}$, and Sec. VI contains a summary.

\section{BASIC DEFINITIONS}

\section{A. Spinors}

The basic philosophy of the $\mathrm{WvdW}$ formalism is to reduce all mathematical objects that belong to higherdimensional representations of the Lorentz group to the twodimensional irreducible representations $D\left(\frac{1}{2}, 0\right)$ and $D\left(0, \frac{1}{2}\right)$. According to these representations, we distinguish covariant and contravariant WvdW spinors $\psi_{A}$ and $\psi^{A}$, respectively. Indices of WvdW spinors are denoted by capital letters throughout. The transition between the two-dimensional representations, which are nonequivalent, is achieved by complex conjugation and a similarity transformation. Complex conjugation is consistently indicated by dotting (undotting) indices, i.e.,

$$
\psi_{\dot{A}}=\left(\psi_{A}\right)^{*}, \quad \psi^{A}=\left(\psi^{\dot{A}}\right)^{*} .
$$

The similarity transformation is mediated by the antisymmetric $2 \times 2$ matrix $\epsilon=i \sigma^{2}$, where $\sigma^{a}(a=1,2,3)$ are the standard Pauli matrices:

$$
\epsilon^{A B}=\epsilon^{\dot{A} \dot{B}}=\epsilon_{A B}=\epsilon_{\dot{A} \dot{B}}=\left(\begin{array}{cc}
0 & +1 \\
-1 & 0
\end{array}\right) .
$$

The matrix $\epsilon$ defines how to raise and lower spinor indices:

$$
\psi^{A}=\epsilon^{A B} \psi_{B}, \quad \psi^{\dot{A}}=\epsilon^{\dot{A} \dot{B}} \psi_{\dot{B}}, \quad \psi_{A}=\psi^{B} \epsilon_{B A}, \quad \psi_{\dot{A}}=\psi^{\dot{B}} \psi_{\dot{B} \dot{A}} .
$$

Moreover, $\epsilon$ provides the set of Clebsch-Gordan coefficients that project the product representations $D\left(\frac{1}{2}, 0\right) \otimes D\left(\frac{1}{2}, 0\right)$ and
$D\left(0, \frac{1}{2}\right) \otimes D\left(0, \frac{1}{2}\right)$ onto the trivial representation. For this reason, it is possible to define a Lorentz-invariant spinor product through

$$
\begin{gathered}
\langle\phi \psi\rangle=\phi_{A} \psi^{A}=\phi_{1} \psi_{2}-\phi_{2} \psi_{1}, \\
\langle\phi \psi\rangle^{*}=\phi_{\dot{A}} \psi^{\dot{A}}=\left(\phi_{1} \psi_{2}-\phi_{2} \psi_{1}\right)^{*},
\end{gathered}
$$

and $\epsilon$ is called a spinor metric. By definition, the spinor product is antisymmetric:

$$
\langle\phi \psi\rangle=-\langle\psi \phi\rangle, \quad\langle\phi \phi\rangle=0 .
$$

The fact that an antisymmetric tensor built of twodimensional objects cannot have a rank higher than 2 implies a Schouten identity for the spinor metric,

$$
\epsilon^{A B} \epsilon^{C D}+\epsilon^{A C} \epsilon^{D B}+\epsilon^{A D} \epsilon^{B C}=0,
$$

which in terms of spinor products reads

$$
\langle\phi \psi\rangle\langle\xi \eta\rangle+\langle\phi \xi\rangle\langle\eta \psi\rangle+\langle\phi \eta\rangle\langle\psi \xi\rangle=0 .
$$

\section{B. Four-vectors}

Minkowski four-vectors belong to the representation $D\left(\frac{1}{2}, \frac{1}{2}\right)=D\left(\frac{1}{2}, 0\right) \otimes D\left(0, \frac{1}{2}\right)$ of the Lorentz group. The transition of the usual form of a four-vector $k^{\mu}=\left(k^{0}, \mathbf{k}\right)$ to the spinor representation $D\left(\frac{1}{2}, \frac{1}{2}\right)$ is provided by the matrices

$$
\sigma^{\mu, \dot{A} B}=\left(\sigma^{0}, \boldsymbol{\sigma}\right), \quad \sigma_{A \dot{B}}^{\mu}=\left(\sigma^{0},-\boldsymbol{\sigma}\right),
$$

consisting of the two-dimensional unit matrix $\sigma^{0}$ and the Pauli matrices $\sigma^{a}$. Each four-vector $k^{\mu}$ is related to a $2 \times 2$ matrix

$$
K_{\dot{A} B}=k^{\mu} \sigma_{\mu, \dot{A} B}=\left(\begin{array}{cc}
k^{0}+k^{3} & k^{1}+i k^{2} \\
k^{1}-i k^{2} & k^{0}-k^{3}
\end{array}\right),
$$

which is Hermitian if the components of $k^{\mu}$ are real. The rules for dotting, undotting, raising, and lowering spinor indices also apply to the indices of the $\sigma$ matrices; in particular, we have

$$
\sigma_{\dot{A} B}^{\mu}=\sigma^{\mu, \dot{C} D} \epsilon_{\dot{C} \dot{A}} \epsilon_{D B}, \quad \sigma_{A \dot{B}}^{\mu}=\left(\sigma_{\dot{A} B}^{\mu}\right)^{*} .
$$

We note that the coefficients of the transpose $K^{T}$ of a matrix $K$ read $K_{B A}$ if the ones of $K$ are denoted by $K_{\dot{A} B}$; i.e., transposing a matrix interchanges the spinor indices without moving the position of the overdot. Thus the Hermiticity of the $\sigma$ matrices is expressed by

$$
\sigma^{\mu, \dot{A} B}=\sigma^{\mu, B \dot{A}}, \quad \sigma_{A \dot{B}}^{\mu}=\sigma_{\dot{B} A}^{\mu} .
$$

The $\sigma$ matrices obey the important relations

$$
\begin{aligned}
\sigma_{\dot{A} B}^{\mu} \sigma^{\nu, \dot{A} B}= & 2 g^{\mu \nu}, \quad \sigma_{\dot{A} B}^{\mu} \sigma^{\nu, \dot{A} C}+\sigma_{\dot{A} B}^{\nu} \sigma^{\mu, \dot{A} C}=2 g^{\mu \nu} \delta_{B}^{C}, \\
& \sigma_{\dot{A} B}^{\mu} \sigma_{\mu, \dot{C} D}=2 \epsilon_{\dot{A} \dot{C}} \epsilon_{B D} .
\end{aligned}
$$


The first of these relations translates the Minkowski inner product of 2 four-vectors $k^{\mu}$ and $p^{\mu}$ into

$$
2 k \cdot p=k_{\mu} 2 g^{\mu \nu} p_{\nu}=k_{\mu} \sigma_{\dot{A} B}^{\mu} \sigma^{\nu, \dot{A} B} p_{\nu}=K_{\dot{A} B} P^{\dot{A} B},
$$

and the second one implies

$$
K_{\dot{A} B} K^{\dot{A} C}=k^{2} \delta_{B}^{C} .
$$

In order to reduce terms involving a matrix $K_{\dot{A} B}$ to spinor products, it is necessary to express $K_{\dot{A} B}$ in terms of spinors. For a real four-vector, the matrix $K_{\dot{A} B}$ is Hermitian and can be decomposed into its eigenvectors $n_{i, A}(i=1,2)$ and eigenvalues $\lambda_{i}$ :

$$
\begin{aligned}
& K_{\dot{A} B}=\sum_{i=1,2} \lambda_{i} n_{i, \dot{A}} n_{i, B}, \quad \lambda_{1,2}=k^{0} \pm|\mathbf{k}|, \\
& n_{1, A}=\left(\begin{array}{c}
e^{-i \phi} \cos \frac{\theta}{2} \\
\sin \frac{\theta}{2}
\end{array}\right), \quad n_{2, A}=\left(\begin{array}{c}
\sin \frac{\theta}{2} \\
-e^{+i \phi} \cos \frac{\theta}{2}
\end{array}\right) \text {, }
\end{aligned}
$$

where $\theta$ and $\phi$ denote the polar and azimuthal angles of $\mathbf{k}$ $=|\mathbf{k}| \mathbf{e}$, respectively:

$$
\mathbf{e}=\left(\begin{array}{c}
\cos \phi \sin \theta \\
\sin \phi \sin \theta \\
\cos \theta
\end{array}\right) .
$$

For timelike vectors $\left(k^{2}>0\right)$, it is often convenient to include the eigenvalues $\lambda_{i}$ in the normalization of the eigenvectors, resulting in

$$
K_{\dot{A} B}=\sum_{i=1,2} \kappa_{i, \dot{A}} \kappa_{i, B}, \quad \kappa_{i, A}=\sqrt{\lambda_{i}} n_{i, A} .
$$

The phases of $n_{i, A}$ are chosen such that the orthonormality relations read

$$
\left\langle n_{i} n_{i}\right\rangle=0, \quad\left\langle n_{2} n_{1}\right\rangle=-\left\langle n_{1} n_{2}\right\rangle=+1 .
$$

Note also the relations for the eigenvalues $\lambda_{i}$ :

$$
\operatorname{det}\left(K_{\dot{A} B}\right)=\lambda_{1} \lambda_{2}=k^{2}, \quad\left\langle\kappa_{2} \kappa_{1}\right\rangle=\sqrt{\lambda_{1} \lambda_{2}}=\sqrt{k^{2}} .
$$

The special case of a lightlike vector $\left(k^{2}=0\right)$ is of particular importance. In this case the eigenvalue $\lambda_{2}$ of Eqs. (2.15) vanishes, and the matrix $K_{\dot{A} B}$ factorizes into a single product of two spinors:

$$
\begin{gathered}
K_{\dot{A} B}=k^{\mu} \sigma_{\mu, \dot{A} B}=k_{\dot{A}} k_{B}, \\
k_{A}=\sqrt{2 k^{0}} n_{1, A}=\sqrt{2 k^{0}}\left(\begin{array}{c}
e^{-i \phi} \cos \frac{\theta}{2} \\
\sin \frac{\theta}{2}
\end{array}\right) .
\end{gathered}
$$

In this context, $k_{A}$ is called a momentum spinor.

Finally, we remark that the decomposition (2.15) is a very convenient, but not unique, possibility to express a fourvector $k^{\mu}$ with $k^{2} \neq 0$ in terms of $\mathrm{WvdW}$ spinors. Any splitting of $k^{\mu}$ into two lightlike four-vectors yields a decomposition into spinors, since lightlike vectors factorize, as seen above. For instance, choosing an arbitrary lightlike fourvector $a^{\mu}\left(a^{2}=0\right)$ with $a \cdot k>0$ and defining

$$
\alpha=\frac{k^{2}}{2 a \cdot k}, \quad b^{\mu}=k^{\mu}-\alpha a^{\mu},
$$

yields a possible decomposition $k^{\mu}=\alpha a^{\mu}+b^{\mu}$. In terms of WvdW spinors, this corresponds to an arbitrarily chosen spinor $a_{A}$ with $K_{\dot{C} D} a^{\dot{C}} a^{D}>0$, leading to the decomposition

$$
K_{\dot{A} B}=\alpha a_{\dot{A}} a_{B}+b_{\dot{A}} b_{B},
$$

with

$$
b_{A}=-\frac{K_{\dot{B A}} a^{\dot{B}}}{\sqrt{K_{\dot{C}{ } a^{\dot{C}} a^{D}}}}, \quad \alpha=\frac{k^{2}}{K_{\dot{C} D} a^{\dot{C}} a^{D}} .
$$

\section{WAVE FUNCTIONS FOR HELICITY EIGENSTATES}

\section{A. Spin- $\frac{1}{2}$ particles}

Dirac spinors $\Psi$ belong to the representation $D\left(\frac{1}{2}, 0\right)$ $\oplus D\left(0, \frac{1}{2}\right)$ of the Lorentz group. Thus, in terms of $\mathrm{WvdW}$ spinors, they are represented by

$$
\Psi=\left(\begin{array}{c}
\phi_{A} \\
\psi^{\dot{A}}
\end{array}\right) .
$$

The WvdW formalism consistently employs the chiral representation of the Dirac matrices $\gamma^{\mu}$ :

$$
\gamma^{\mu}=\left(\begin{array}{cc}
0 & \sigma_{A \dot{B}}^{\mu} \\
\sigma^{\mu, \dot{A} B} & 0
\end{array}\right), \quad \gamma^{5}=i \gamma^{0} \gamma^{1} \gamma^{2} \gamma^{3}=\left(\begin{array}{cc}
\sigma^{0} & 0 \\
0 & -\sigma^{0}
\end{array}\right) .
$$

We are interested in plane-wave solutions $\Psi=$ $\exp \{\mp i k x\} \Psi_{k}^{( \pm)}$of Dirac's equation

$$
(i \not b-m) \Psi=0,
$$

which describe the propagation of free Dirac fermions and antifermions, respectively. Inserting Eqs. (3.1) and (3.2) into Dirac's equation, we obtain the coupled pair of Weyl equations

$$
\begin{array}{r}
K_{A \dot{B}} \psi_{k}^{( \pm), \dot{B}}= \pm m \phi_{k, A}^{( \pm)}, \quad K^{\dot{A} B} \phi_{k, B}^{( \pm)}= \pm m \psi_{k}^{( \pm), \dot{A}}, \\
k^{2}=m^{2} .
\end{array}
$$

Upon applying the decomposition (2.17) of $K_{\dot{A} B}$, the following solutions can be easily constructed: 


$$
\Psi_{k, 1}^{( \pm)}=\left(\begin{array}{c}
\kappa_{1, A} \\
\mp \kappa_{2}^{\dot{A}}
\end{array}\right), \quad \Psi_{k, 2}^{( \pm)}=\left(\begin{array}{c} 
\pm \kappa_{2, A} \\
\dot{A} \\
\kappa_{1}
\end{array}\right)
$$

The corresponding adjoint spinors $\bar{\Psi}=\Psi^{\dagger} \gamma_{0}$ read

$$
\bar{\Psi}_{k, 1}^{( \pm)}=\left(\mp \kappa_{2}^{A}, \kappa_{1, \dot{A}}\right), \quad \Psi_{k, 2}^{( \pm)}=\left(\kappa_{1}^{A}, \pm \kappa_{2, \dot{A}}\right) .
$$

The solutions obey the standard normalization

$$
\Psi_{k, i}^{( \pm) \dagger} \Psi_{k, j}^{(\tau)}=2 k^{0} \delta_{\tau_{ \pm}} \delta_{i j}, \quad \Psi_{k, i}^{( \pm)} \Psi_{k, j}^{(\tau)}= \pm 2 m \delta_{\tau_{ \pm}} \delta_{i j}
$$

Moreover, it is straightforward to check that they indeed form eigenstates of the helicity projector

$$
\Sigma_{k}^{ \pm}=\frac{1}{2}\left(1 \pm \gamma^{5} k_{k}\right), \quad s_{k}^{\mu}=\frac{k^{0}}{m} \frac{k^{\mu}}{|\mathbf{k}|}-g^{\mu 0} \frac{m}{|\mathbf{k}|} .
$$

More precisely, $\Sigma_{k}^{+}$projects onto $\Psi_{k, 1}^{(+)}$and $\Psi_{k, 2}^{(-)}, \Sigma_{k}^{-}$onto $\Psi_{k, 1}^{(-)}$and $\Psi_{k, 2}^{(+)}$; i.e., $\Psi_{k, 1}^{(+)}$describes a right-handed fermion, $\Psi_{k, 2}^{(-)}$a right-handed antifermion, etc.

For massless fermions the Weyl equations (3.4) decouple, and the helicity eigenstates take the simple form

$$
\begin{gathered}
\Psi_{k, 1}^{( \pm)}=\left(\begin{array}{c}
k_{A} \\
0
\end{array}\right), \quad \Psi_{k, 2}^{( \pm \pm)}=\left(\begin{array}{c}
0 \\
k^{\dot{A}}
\end{array}\right), \\
\Psi_{k, 1}^{( \pm)}=\left(0, k_{\dot{A}}\right), \quad \Psi_{k, 2}^{( \pm)}=\left(k^{A}, 0\right) .
\end{gathered}
$$

For the decomposition (2.22) of $K_{\dot{A} B}$, also simple planewave solutions exist, namely,

$$
\Psi_{k, 1}^{( \pm)}=\left(\begin{array}{c}
b_{A} \\
\mp \sqrt{\alpha} a^{\dot{A}}
\end{array}\right), \quad \Psi_{k, 2}^{( \pm)}=\left(\begin{array}{c} 
\pm \sqrt{\alpha} a_{A} \\
b^{\dot{A}}
\end{array}\right),
$$

which are, however, not related to definite helicity states.

\section{B. Massive spin-1 particles}

Spin-1 fields $V^{\mu}$ transform like ordinary four-vectors under Lorentz transformations. This means that polarization vectors $\varepsilon^{\mu}$ for such fields can be related to $2 \times 2$ matrices $\varepsilon_{\dot{A} B}$ in the same way as described in Eq. (2.9) for a general four-vector $k^{\mu}$. However, one should keep in mind that polarization vectors need not be real so that $\varepsilon_{\dot{A} B}$ is not Hermitian in general.

The free field $V^{\mu}$ of a massive vector particle is governed by Proca's equation

$$
\left[\left(\partial^{2}+m^{2}\right) g^{\mu \nu}-\partial^{\mu} \partial^{\nu}\right] V_{\nu}=0
$$

which for $m \neq 0$ is equivalent to the Klein-Gordon equation, in conjunction with the transversality condition:

$$
\left(\partial^{2}+m^{2}\right) V^{\mu}=0, \quad \partial \cdot V=0 .
$$

With the ansatz $V^{\mu}=\exp \{\mp i k x\} \varepsilon_{\mu}(k)$ for particles and antiparticles, respectively, we arrive at

$$
k^{2}=m^{2}, \quad k^{\nu} \varepsilon_{\nu}(k)=\frac{1}{2} K^{\dot{A} B} \varepsilon_{\dot{A} B}(k)=0 .
$$

For $m \neq 0$ there are three linearly independent, spacelike polarization vectors $\varepsilon_{i}^{\mu}(k)$, which are usually orthonormalized according to

$$
\varepsilon_{i}^{\mu}(k) \varepsilon_{j, \mu}^{*}(k)=\frac{1}{2} \varepsilon_{i, \dot{A} B}(k) \varepsilon_{j}^{* \dot{A} B}(k)=-\delta_{i j}, \quad i, j=0, \pm,
$$

where $\varepsilon^{* \dot{A} B}=\varepsilon_{\mu}^{*} \sigma^{\mu, \dot{A} B}$. A helicity basis for the $\varepsilon_{i}^{\mu}(k)$ is given by

$$
\begin{aligned}
\varepsilon_{ \pm}^{\mu}(k)= & \frac{e^{\mp i \phi}}{\sqrt{2}}(0,-\cos \theta \cos \phi \pm i \sin \phi \\
& -\cos \theta \sin \phi \mp i \cos \phi, \sin \theta),
\end{aligned}
$$

$\varepsilon_{0}^{\mu}(k)=s_{k}^{\mu}=\frac{k^{0}}{m}\left(\frac{|\mathbf{k}|}{k^{0}}, \cos \phi \sin \theta, \sin \phi \sin \theta, \cos \theta\right)$,

where $s_{k}^{\mu}$ is related to $k^{\mu}$ as given in Eqs. (3.8). In terms of WvdW spinors, this spin basis reads

$$
\begin{aligned}
\varepsilon_{+, \dot{A} B}(k) & =\sqrt{2} n_{2, \dot{A}} n_{1, B}, \quad \varepsilon_{-, \dot{A} B}(k)=\sqrt{2} n_{1, \dot{A}} n_{2, B}, \\
\varepsilon_{0, \dot{A} B}(k) & =\frac{1}{m}\left(\kappa_{1, \dot{A}} \kappa_{1, B}-\kappa_{2, \dot{A}} \kappa_{2, B}\right),
\end{aligned}
$$

where the spinors $n_{i, A}$ and $\kappa_{i, A}$ are defined in Eqs. (2.15) and (2.17), respectively. Note that care has to be taken when dealing with conjugate polarization vectors, which, in particular, occur for outgoing spin-1 particles in transition amplitudes. In such cases the transition from the four-vector $\varepsilon^{* \mu}$ to the $2 \times 2$ matrix $\varepsilon_{\dot{A} B}^{*}$ upon contraction with the $\sigma$ matrices has to be consistently performed for the conjugated polarization vector, i.e., $\varepsilon_{\dot{A} B}^{*}=\varepsilon_{\mu}^{*} \sigma_{\dot{A} B}^{\mu}$, which in general is different from taking the complex conjugate of $\varepsilon_{\dot{A} B}$. For clarity, we give also the helicity basis for an outgoing spin-1 particle:

$$
\begin{aligned}
& \varepsilon_{+, \dot{A} B}^{*}(k)=\sqrt{2} n_{1, \dot{A}} n_{2, B}, \quad \varepsilon_{-, \dot{A} B}^{*}(k)=\sqrt{2} n_{2, \dot{A}} n_{1, B}, \\
& \varepsilon_{0, \dot{A} B}^{*}(k)=\frac{1}{m}\left(\kappa_{1, \dot{A}} \kappa_{1, B}-\kappa_{2, \dot{A}} \kappa_{2, B}\right),
\end{aligned}
$$

which correspond to the conjugate polarization vectors $\varepsilon_{i}^{*}$ of $\varepsilon_{i}$ given in Eqs. (3.15). From Eqs. (3.16) and (3.17), we obtain the relations

$$
\varepsilon_{i, \dot{A} B}(k)=\varepsilon_{-i, B \dot{A}}(k)=\varepsilon_{-i, \dot{A} B}^{*}(k)=\varepsilon_{i, B \dot{A}}^{*}(k) .
$$

Finally, we give a simple spin basis for the decomposition (2.22) of $K_{A B}$, 
TABLE I. Prescriptions for translating ordinary Feynman rules for external fields and propagators into the WvdW formalism.

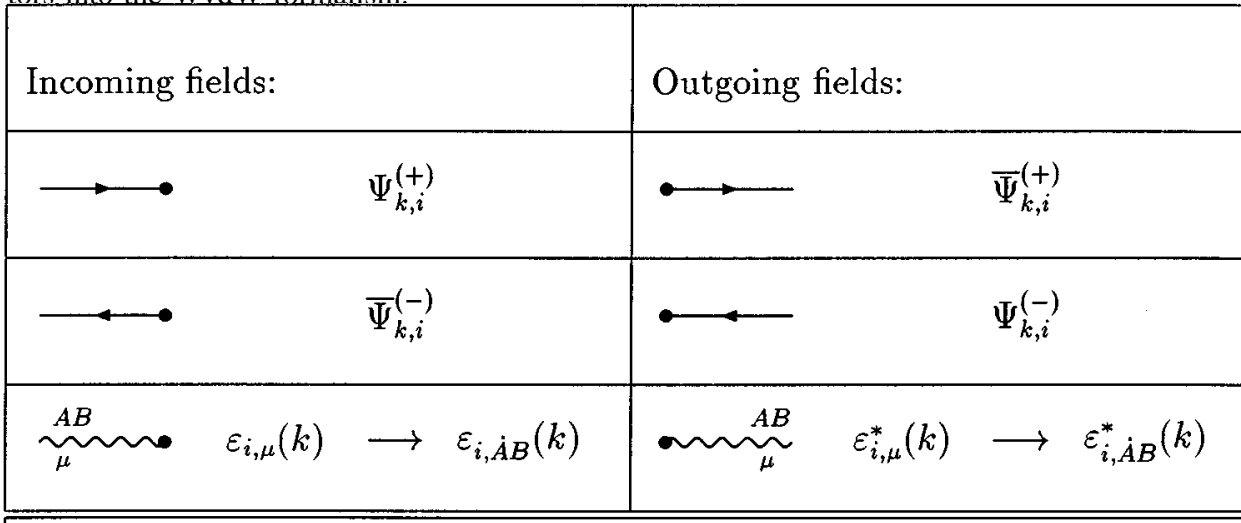

\section{Propagators:}

\begin{tabular}{|c|c|c|c|c|c|}
\hline$\stackrel{B \quad}{\longrightarrow} \quad A$ & $\frac{i\left(\not k+m_{f}\right)}{k^{2}-m_{f}^{2}}$ & $\longrightarrow$ & $\frac{i}{k^{2}-m_{f}^{2}}$ & $\begin{array}{c}m_{f} \delta_{A}^{B} \\
K^{\dot{A} B}\end{array}$ & $\left.\begin{array}{c}K_{A \dot{B}} \\
m_{f} \delta_{\dot{B}}^{\dot{A}}\end{array}\right)$ \\
\hline$\overbrace{\mu}^{A B} \sim_{\nu}^{C D}$ & $\frac{-i g_{\mu \nu}}{k^{2}-M_{V}^{2}}$ & $\longrightarrow$ & $\frac{-2 i \epsilon_{\dot{A} \dot{C}} \epsilon_{1}}{k^{2}-M}$ & & \\
\hline
\end{tabular}

$$
\begin{gathered}
\varepsilon_{+, \dot{A} B}(p)=\frac{\sqrt{2} a_{\dot{A}} b_{B}}{\langle a b\rangle^{*}}, \quad \varepsilon_{-, \dot{A} B}(p)=\frac{\sqrt{2} b_{\dot{A}} a_{B}}{\langle a b\rangle}, \\
\varepsilon_{0, \dot{A} B}(p)=\frac{1}{m}\left(b_{\dot{A}} b_{B}-\alpha a_{\dot{A}} a_{B}\right),
\end{gathered}
$$

which is, however, not related to helicity eigenstates.

\section{Massless spin-1 particles}

In contrast with the case of spin- $\frac{1}{2}$ particles, the zero-mass limit for vector bosons confronts us with a new physical situation. For $m \rightarrow 0$ the polarization vector $\varepsilon_{0}^{\mu}$ does not exist, since the longitudinal polarization does not yield a physical state for massless spin-1 particles. The corresponding degree of freedom manifests itself in the arbitrariness of gauge for the two polarization vectors $\varepsilon_{ \pm}^{\mu}$. In the language of WvdW spinors, this fact is expressed very elegantly:

$$
\begin{aligned}
& \varepsilon_{+, \dot{A} B}(k)=\frac{\sqrt{2} g_{+, \dot{A}} k_{B}}{\left\langle g_{+} k\right\rangle^{*}}, \quad \varepsilon_{-, \dot{A} B}(k)=\frac{\sqrt{2} k_{\dot{A}} g_{-, B}}{\left\langle g_{-} k\right\rangle}, \\
& \varepsilon_{+, \dot{A} B}^{*}(k)=\frac{\sqrt{2} k_{\dot{A}} g_{+, B}}{\left\langle g_{+} k\right\rangle}, \quad \varepsilon_{-, \dot{A} B}^{*}(k)=\frac{\sqrt{2} g_{-, \dot{A}} k_{B}}{\left\langle g_{-} k\right\rangle^{*}},
\end{aligned}
$$

where $g_{ \pm, A}$ denote arbitrary spinors with $\left\langle g_{ \pm} k\right\rangle \neq 0$; they are called gauge spinors. The difference of two matrices $\varepsilon_{i, \dot{A} B}(k)$ for differently chosen gauge spinors is proportional to the momentum matrix $k_{A} k_{B}$, as can be easily checked by applying the Schouten identity (2.6). The transversality condition $k \cdot \varepsilon_{ \pm}=0$ is still fulfilled for any $g_{ \pm, A}$; i.e., the Lorentz gauge is maintained for the $\varepsilon_{ \pm}$of Eqs. (3.20). Transi- tion amplitudes do not depend on the choice of gauge spinors. This means that the $g_{ \pm, A}$ can be appropriately chosen to simplify the algebraic expression. Note that it is even possible to take different sets of gauge spinors for each helicity channel.

\section{CALCULATION OF HELICITY AMPLITUDES}

\section{A. Feynman rules}

Feynman rules are usually written down in terms of fourvectors and Dirac matrices. Here we describe how such Feynman rules can be directly translated into the language of WvdW spinors. Obviously, this procedure is much more practical than going back to the underlying Lagrangian and introducing WvdW spinors there.

Given any Feynman graph, the trick is to contract each vector-boson leg of a vertex with the identity $\delta_{v}^{\mu}$ $=\frac{1}{2} \sigma_{\nu}^{\dot{A} B} \sigma_{\dot{A} B}^{\mu}$ and to shift the factor $\sigma_{\dot{A} B}^{\mu}$ to the vector-boson propagator or to the external wave function of the vector boson that is attached to this vertex. Moreover, for each occurring Dirac matrix, the chiral representation (3.2) has to be used. In particular, the unit matrix 1 in the Dirac space and a slashed quantity $d$ read

$$
\mathbf{1}=\left(\begin{array}{cc}
\delta_{A}^{B} & 0 \\
0 & \delta_{\dot{B}}^{\dot{A}}
\end{array}\right), \quad d \boldsymbol{d}=\left(\begin{array}{cc}
0 & a_{A \dot{B}} \\
a^{\dot{A} B} & 0
\end{array}\right),
$$

respectively. Tables I and II summarize the necessary changes of the generic Feynman rules for the electroweak standard model in the 't Hooft-Feynman gauge. The explicit values for the couplings can, for instance, be found in Ref. 
TABLE II. Prescriptions for translating ordinary Feynman rules for vertices into the WvdW formalism. Scalar fields, fermion fields, and vector fields are generically denoted by $S, f$, and $V$, respectively, and $\omega_{ \pm}$ $=\frac{1}{2}\left(1 \pm \gamma^{5}\right)$ are the chirality projectors.

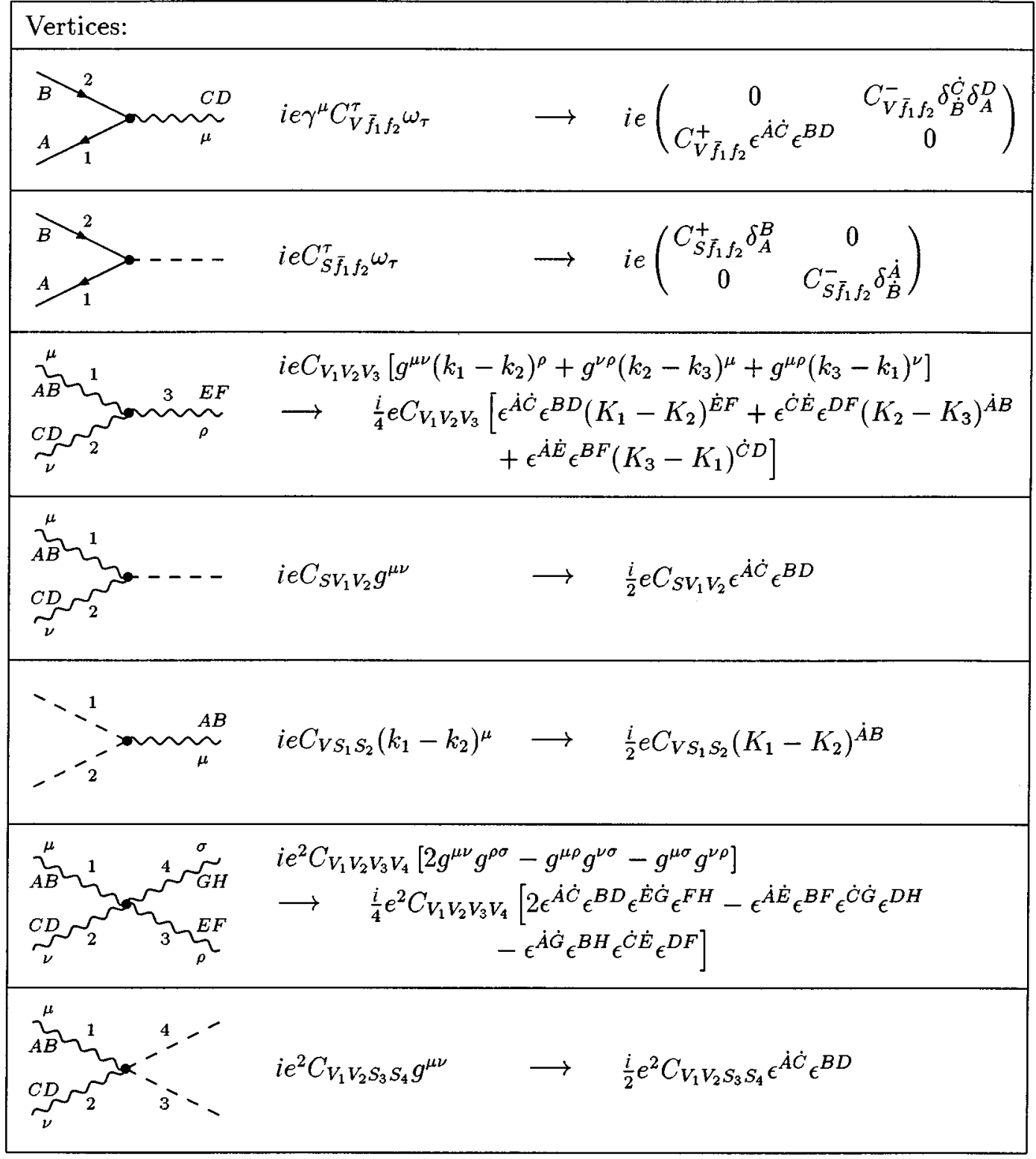

[12]. The new Feynman rules for other conventions or different models can easily be worked out by the reader.

Once the Feynman rules are settled, it is very easy to explicitly write down helicity amplitudes for any process at the tree level. Expressing all particle momenta in terms of spinors, as described in Sec. II B, each helicity amplitude is reduced to an algebraic expression in terms of antisymmetric spinor products $\langle\phi \psi\rangle$ after all spinor indices are contracted. These spinor contractions can be performed like usual contractions of Lorentz indices, apart from taking care of the antisymmetry. The form of the amplitudes obtained this way is already well suited to numerical evaluations.

\section{B. Discrete symmetries}

Discrete symmetries relate helicity amplitudes of different processes or of one and the same process. Thus they either provide convenient cross-checks for results or they allow for a reduction of the algebraic work by generating various amplitudes from a generic set of amplitudes. In the following we show how to derive the relations implied by crossing symmetry, parity, and $C P$ symmetry, which are the most important discrete symmetries in practice.

(i) Crossing symmetry. Crossing symmetry transforms an incoming particle into the corresponding outgoing antiparticle or vice versa. Denoting the momentum of a given particle by $k$, the inversion $k \rightarrow-k$ can be consistently obtained by substituting

$$
\kappa_{i, \dot{A}} \rightarrow-\kappa_{i, \dot{A}}, \quad \kappa_{i, A} \rightarrow+\kappa_{i, A}
$$

in the decomposition (2.17) of $k$, i.e., by inverting the contravariant parts only. In order to relate helicity amplitudes, it 
is also necessary to consider the relation between the wave functions of the respective incoming and outgoing fields. Inspecting the explicit form of the wave functions for the helicity eigenstates of Sec. III, one finds that the substitution (4.2) transforms incoming (outgoing) particles into outgoing (incoming) antiparticles with reversed helicity modulo sign change. Specifically, there is a global factor -1 for each crossed spin-1 field and a factor $\pm \operatorname{sgn}(\sigma)$ for each incomingoutgoing fermion with helicity $\sigma$ that is involved in the crossing.

(ii) Parity. If parity is a symmetry, every helicity amplitude, up to a phase factor, agrees with the corresponding amplitude with opposite helicities, after the spatial parts of all momenta are inverted. At the level of WvdW spinors, the inversion of the spatial parts of momenta is connected with the interchange of the spinors $\psi_{A}$ and $\psi^{A}$. It turns out that each helicity amplitude, up to a global sign factor, agrees with the complex conjugate amplitude with opposite helicity configuration. More precisely, if $i \mathcal{M}\left(\sigma_{i} ; \lambda_{j}\right)$ is an amplitude involving $n$ fermions and $\bar{n}$ antifermions with helicities $\sigma_{i}$, and $n_{V}$ vector bosons with helicities $\lambda_{j}$, we get the relations

$$
\mathcal{M}\left(-\sigma_{i} ;-\lambda_{j}\right)=(-1)^{\bar{n}} \operatorname{sgn}\left(\sigma_{1} \cdots \sigma_{n+\bar{n}}\right) \mathcal{M}\left(\sigma_{i} ; \lambda_{j}\right)^{*}
$$

for tree-level amplitudes. If parity is violated, these relations for amplitudes remain valid if left- and right-handed couplings are appropriately substituted. The explicit derivation of Eq. (4.3) and the modifications for broken parity can be found in the Appendix.

(iii) $C P$ symmetry. $C P$ is the product of parity, which is explained above, and charge conjugation, which interchanges particles with the respective antiparticles. Therefore, $C P$ symmetry leads to relations between the helicity amplitudes of a given process and the complex conjugate helicity amplitudes with reversed helicities of the process involving the respective antiparticles. We consider an amplitude $i \mathcal{M}\left(\sigma_{i} ; \lambda_{j}\right)$ and the one for the $C P$-related process, $i \overline{\mathcal{M}}\left(\sigma_{i} ; \lambda_{j}\right)$, where the respective helicities for the fermions and vector bosons are assigned as above. If $C P$ is an exact symmetry, these two matrix elements are related by

$$
\begin{aligned}
\overline{\mathcal{M}}( & \left.-\sigma_{i} ;-\lambda_{j}\right) \\
& =(-1)^{n_{V}+n_{\mathrm{in}}+\bar{n}_{\mathrm{in}}} \operatorname{sgn}\left(\sigma_{1} \cdots \sigma_{n+\bar{n}}\right) \mathcal{M}\left(\sigma_{i} ; \lambda_{j}\right) *
\end{aligned}
$$

at the tree level, where $n_{\text {in }}+\bar{n}_{\text {in }}$ is the total number of incoming fermions and antifermions. The explicit derivation of these relations and their modifications if $C P$ is violated are given in the appendix.

The actual use of these symmetries will be illustrated when we consider helicity amplitudes of concrete processes in Sec. V.

\section{Remarks on the choice of gauge spinors}

Since a helicity amplitude does not depend on the actual insertions for gauge spinors, they can, in principle, be chosen arbitrarily. Usually, a gauge spinor $g_{A}$ is chosen such that as

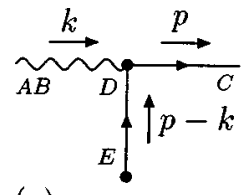

(a)

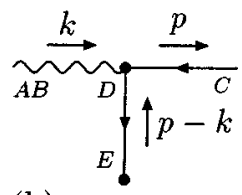

(b)
FIG. 1. Subdiagrams containing the fermion propagator denominator $\left[(p-k)^{2}-m_{f}^{2}\right]^{-1}$ for processes with an incoming photon of momentum $k\left(k^{2}=0\right)$ and an outgoing fermion or antifermion of momentum $p\left(p^{2}=m_{f}^{2}\right)$.

many terms as possible in an amplitude vanish. However, as can be seen from Eqs. (3.20), an arbitrary choice of $g_{A}$ in general leads to unphysical poles at the zeros of $\langle g k\rangle$ in individual terms that contribute to an amplitude. Of course, the unphysical pole drops out in the final result, but this cancellation can cause instabilities in a numerical evaluation.

Unphysical poles can, for instance, consistently be avoided by setting $g_{A}=n_{2, A}$, where $n_{2, A}$ is related to the momentum $k$ of the massless spin-1 particle as specified in Eqs. (2.15). This choice is identical with Eqs. (3.16) for the transverse modes of a massive spin-1 particle. The drawback of this choice is that no algebraic simplifications result.

Another possibility to avoid numerical problems that are due to unphysical poles is to cancel such poles analytically before the numerical evaluation. In general, this task can be extremely cumbersome, and the additional work devalues preceding simplifications. In the following we give a very convenient choice of $g_{A}$, yielding desirable simplifications without leaving uncanceled unphysical poles.

Consider a process with an incoming photon of momentum $k$ and an outgoing fermion $f$ of momentum $p$; the momentum matrix for $p$ is denoted by

$$
P_{\dot{A} B}=\sum_{i=1,2} \kappa_{i, \dot{A}} \kappa_{i, B} .
$$

We choose the same gauge spinor

$$
g_{A}=P_{A \dot{B}} k^{\dot{B}}=\sum_{i=1,2} \kappa_{i, A}\left\langle\kappa_{i} k\right\rangle^{*}
$$

for both photon helicities. The problematic denominators contained in the photon polarization vectors (3.20) are given by

$$
\langle g k\rangle=k^{A} P_{A \dot{B}} k^{\dot{B}}=2(p \cdot k) .
$$

This has the form of the inverse propagator $(p-k)^{2}-m_{f}^{2}=$ $-2(p \cdot k)$ that appears if the incoming photon directly couples to the outgoing fermion $f$ so that no unphysical pole is introduced at all. The subdiagram containing this propagator is shown in Fig. 1(a). In Feynman graphs that contain this subdiagram, we obtain the factor $(p \cdot k)^{-2}$; one power is due to the propagator, and another is due to the polarization vector. For small $(p \cdot k)$ we again get an unwanted cancellation of one power in $(p \cdot k)$. However, this cancellation can be easily performed analytically. Denoting the Dirac spinor of the outgoing fermion with helicity $\sigma$, generically, by 


$$
\bar{\Psi}_{p, i}^{(+)}=\left(\psi^{C}, \phi_{\dot{C}}\right)
$$

with

$$
(\phi, \psi)=\left\{\begin{array}{lll}
\left(\kappa_{1},-\kappa_{2}\right) & \text { for } i=1 & (\sigma=+), \\
\left(\kappa_{2}, \kappa_{1}\right) & \text { for } i=2 & (\sigma=-),
\end{array}\right.
$$

and using the wave function $\varepsilon_{\dot{A} B}$ for the photon with helicity $\lambda$, the subdiagram reads

$$
\begin{aligned}
T_{\lambda}^{\gamma f}(\sigma)= & -\frac{Q_{f} e}{2(p \cdot k)}\left(\psi^{C}, \phi_{\dot{C}}\right)\left(\begin{array}{cc}
0 & \delta_{D}^{\dot{A}} \delta_{C}^{B} \\
\epsilon^{\dot{A}}{ }^{B} \epsilon^{B D} & 0
\end{array}\right) \\
& \times \varepsilon_{\dot{A} B}\left(\begin{array}{cc}
m_{f} \delta_{D}^{E} & (p-k)_{D \dot{E}} \\
(p-k)^{\dot{D} E} & m_{f} \delta_{\dot{E}}^{\dot{D}}
\end{array}\right),
\end{aligned}
$$

where $Q_{f}$ is the relative electromagnetic charge of the fermion $f$. Upon inserting Eq. (4.5), Eq. (4.6), and the explicit expressions (3.20) for $\varepsilon_{+, \dot{A} B}$, we get

$$
\begin{aligned}
T_{+}^{\gamma f}(\sigma)= & \frac{Q_{f} e}{2 \sqrt{2}(p \cdot k)^{2}}\langle k \psi\rangle \\
& \times\left(2(p \cdot k) k^{E}+m_{f}\left[\langle\phi \psi\rangle k^{E}-\langle k \psi\rangle \phi^{E}\right.\right. \\
& \left.\left.+\langle k \phi\rangle \psi^{E}\right], 0\right) .
\end{aligned}
$$

Here we have used that $P_{\dot{A} B}=\phi_{\dot{A}} \phi_{B}+\psi_{\dot{A}} \psi_{B}$ and $\langle\phi \psi\rangle$ $=m_{f}$ for both fermion helicities. The expression within square brackets in Eq. (4.10) vanishes according to Schouten's identity (2.6) so that one factor $(p \cdot k)$ cancels. Performing a similar calculation for $T_{-}^{\gamma f}(\sigma)$, we find the simple results

$$
\begin{gathered}
T_{+}^{\gamma f}(\sigma)=\frac{Q_{f} e}{\sqrt{2}(p \cdot k)}\langle k \psi\rangle\left(k^{E}, 0\right), \\
T_{-}^{\gamma f}(\sigma)=\frac{Q_{f} e}{\sqrt{2}(p \cdot k)}\langle k \phi\rangle^{*}\left(0, k_{\dot{E}}\right) .
\end{gathered}
$$

Considering an outgoing antifermion instead of a fermion, as shown in Fig. 1(b), we get

$$
\begin{gathered}
T_{+}^{\gamma \bar{f}}(\sigma)=-\frac{Q_{f} e}{\sqrt{2}(p \cdot k)}\langle k \phi\rangle\left(\begin{array}{c}
k_{E} \\
0
\end{array}\right), \\
T_{-}^{\gamma \bar{f}}(\sigma)=-\frac{Q_{f} e}{\sqrt{2}(p \cdot k)}\langle k \psi\rangle *\left(\begin{array}{c}
0 \\
\dot{E}
\end{array}\right),
\end{gathered}
$$

where the Dirac spinor for the antifermion is generically denoted by

$$
\Psi_{p, i}^{(-)}=\left(\begin{array}{c}
\phi_{C} \\
\dot{C}
\end{array}\right)
$$

with

$$
(\phi, \psi)=\left\{\begin{array}{llll}
\left(\kappa_{1}, \kappa_{2}\right) & \text { for } & i=1 & (\sigma=-), \\
\left(-\kappa_{2}, \kappa_{1}\right) & \text { for } & i=2 & (\sigma=+) .
\end{array}\right.
$$

From the results (4.11) and (4.12) for diagrams (a) and (b) of Fig. 1, respectively, all similar diagrams involving outgoing photons and/or incoming (anti)fermions follow by crossing, as described in the previous section.

The above procedure also works if the (anti)fermions in Fig. 1 are replaced by charged gauge bosons, e.g., by the $W$ boson of the electroweak standard model. In this case, however, one has to take into account the contribution of the associated would-be Goldstone boson, which appears on internal lines (vertical lines in Fig. 1).

\section{APPLICATIONS}

In order to illustrate the actual use of the formalism described above, we calculate full sets of helicity amplitudes for some processes with massive particles and photons in lowest order. The presented results have been derived analytically and simplified as far as possible; this demonstrates the analytical power of the technique. In addition, the amplitudes have been evaluated by performing the spinor contractions automatically in MATHEMATICA [13], which is very simple. The results of both approaches have been compared numerically.

The following examples only include massive external fermions. For an application of the helicity basis for massive spin-1 particles, we refer to Ref. [7], where the radiative processes $e^{-} \gamma \rightarrow W^{-} \nu_{e} \gamma, e^{-} Z \gamma$ are discussed.

\section{A. Process $\gamma \gamma \rightarrow f \bar{f}$}

For illustration, we start with the simple QED process

$$
\gamma\left(k_{1}, \lambda_{1}\right)+\gamma\left(k_{2}, \lambda_{2}\right) \rightarrow f(p, \sigma)+\bar{f}\left(p^{\prime}, \sigma^{\prime}\right) .
$$

The momentum matrix and the wave function for the outgoing fermion $f$ are denoted as in Eqs. (4.5) and (4.8), respectively. For the outgoing antifermion we define

$$
P_{A B}^{\prime}=\sum_{i=1,2} \kappa_{i, \dot{A}}^{\prime} \kappa_{i, B}^{\prime}
$$

and

$$
\Psi_{p^{\prime}}^{(-)}=\left(\begin{array}{c}
\phi_{C}^{\prime} \\
\psi^{\prime} \dot{C}
\end{array}\right)
$$

with

$$
\left(\phi^{\prime}, \psi^{\prime}\right)= \begin{cases}\left(\kappa_{1}^{\prime}, \kappa_{2}^{\prime}\right) & \text { for } \sigma^{\prime}=-, \\ \left(-\kappa_{2}^{\prime}, \kappa_{1}^{\prime}\right) & \text { for } \sigma^{\prime}=+.\end{cases}
$$

Since we want to make use of the results of the previous section, we set the gauge spinor $g_{n, A}$ for the $n$th photon to

$$
g_{n, A}=P_{A \dot{B}} k_{n}^{\dot{B}}=\sum_{i=1,2} \kappa_{i, A}\left\langle\kappa_{i} k_{n}\right\rangle *, \quad n=1,2 .
$$




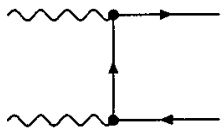

(a)

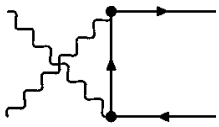

(b)
FIG. 2. Born diagrams for $\gamma \gamma \rightarrow f \bar{f}$.

In lowest order the two Feynman graphs of Fig. 2 contribute. Let us first calculate the contribution $\mathcal{M}_{\text {(a) }}$ of diagram (a) to the full helicity amplitude $\mathcal{M}$. We identify the photon $\gamma(k, \lambda)$ in the subgraph shown in Fig. 1(a) with $\gamma\left(k_{1}, \lambda_{1}\right)$ and denote the contribution of this subgraph by $T_{\lambda_{1}}^{\gamma_{1} f}(\sigma)$. $\mathcal{M}_{\text {(a) }}$ then follows upon multiplication of $T_{\lambda_{1}}^{\gamma_{1} f}(\sigma)$, which is given in Eqs. (4.11), with the second $\gamma f \bar{f}$ vertex and the wave functions for the second photon and the antifermion:

$$
\begin{aligned}
& i \mathcal{M}_{\text {(a) }}\left(\lambda_{1}, \lambda_{2}, \sigma, \sigma^{\prime}\right) \\
& \quad=T_{\lambda_{1}}^{\gamma_{1} f}(\sigma)\left(-i Q_{f} e\right)\left(\begin{array}{cc}
0 & \delta_{\dot{F}}^{\dot{G}} \delta_{E}^{H} \\
\epsilon^{\dot{E} \dot{G}} \epsilon^{F H} & 0
\end{array}\right)\left(\begin{array}{c}
\phi_{F}^{\prime} \\
\psi^{\prime}
\end{array}\right) \varepsilon_{2, \dot{G} H} .
\end{aligned}
$$

Note that we follow the common practice to split off a factor $+i$ from helicity amplitudes throughout. It is trivial to carry out the spinor contractions in Eq. (5.5) for the explicit insertions of $T_{\lambda_{1}}^{\gamma_{1} f}(\sigma)$ and $\varepsilon_{2, \dot{G} H}$. The contribution $\mathcal{M}_{(\mathrm{b})}$ of the diagram in Fig. 2(b) follows from $\mathcal{M}_{\text {(a) }}$ by consistently interchanging the incoming photons. This leads us to the final result for the full helicity amplitudes $\mathcal{M}=\mathcal{M}_{\text {(a) }}+\mathcal{M}_{(\mathrm{b})}$ :

$$
\begin{aligned}
\mathcal{M}\left(+,+, \sigma, \sigma^{\prime}\right)= & \frac{Q_{f}^{2} e^{2} m_{f}}{2\left(p \cdot k_{1}\right)\left(p \cdot k_{2}\right)}\left\langle k_{1} k_{2}\right\rangle^{2}\left\langle\phi \psi^{\prime}\right\rangle^{*}, \\
\mathcal{M}\left(-,-, \sigma, \sigma^{\prime}\right)= & \frac{Q_{f}^{2} e^{2} m_{f}}{2\left(p \cdot k_{1}\right)\left(p \cdot k_{2}\right)}\left(\left\langle k_{1} k_{2}\right\rangle^{*}\right)^{2}\left\langle\phi^{\prime} \psi\right\rangle, \\
\mathcal{M}\left(+,-, \sigma, \sigma^{\prime}\right)= & -\frac{Q_{f}^{2} e^{2}}{2\left(p \cdot k_{1}\right)\left(p \cdot k_{2}\right)}\left\langle k_{2} P k_{1}\right\rangle \\
& \times\left(\left\langle k_{1} \phi^{\prime}\right\rangle\left\langle k_{2} \phi\right\rangle^{*}+\left\langle k_{1} \psi\right\rangle\left\langle k_{2} \psi^{\prime}\right\rangle^{*}\right), \\
\mathcal{M}\left(-,+, \sigma, \sigma^{\prime}\right)= & \left.\mathcal{M}\left(+,-, \sigma, \sigma^{\prime}\right)\right|_{k_{1} \leftrightarrow k_{2}} .
\end{aligned}
$$

Here we have introduced the abbreviation

$$
\left\langle k_{l} P k_{n}\right\rangle=k_{l, \dot{A}} P^{\dot{A} B} k_{n, B}=\sum_{i=1,2}\left\langle k_{l} \kappa_{i}\right\rangle^{*}\left\langle k_{n} \kappa_{i}\right\rangle, \quad l, n=1,2 .
$$

Moreover, we mention that Schouten's identity (2.6) has been used in order to get compact results for $\lambda_{1}=\lambda_{2}= \pm$.
As explained in Sec. IV B, parity relates amplitudes with opposite-helicity configurations through complex conjugation, modulo a sign factor. According to Eq. (4.3), these relations explicitly read

$$
\begin{aligned}
\mathcal{M}\left(-\lambda_{1},-\lambda_{2},\right. & \left.-\sigma,-\sigma^{\prime}\right) \\
& =-\operatorname{sgn}\left(\sigma \sigma^{\prime}\right) \mathcal{M}\left(\lambda_{1}, \lambda_{2}, \sigma, \sigma^{\prime}\right)^{*},
\end{aligned}
$$

consistent with the above results. Alternatively, these relations can be used to generate all helicity amplitudes from the generic results for $\mathcal{M}\left(+,+, \sigma, \sigma^{\prime}\right)$ and $\mathcal{M}\left(+,-, \sigma, \sigma^{\prime}\right)$ given in Eqs. (5.6). The process $\gamma \gamma \rightarrow f \bar{f}$ is also CP symmetric. Taking into account that the $\mathrm{CP}$ transformation interchanges $f$ and $\bar{f}$, Eq. (4.4) implies

$$
\begin{aligned}
\mathcal{M}\left(-\lambda_{1},-\right. & \left.\lambda_{2},-\sigma^{\prime},-\sigma\right) \\
& =\left.\operatorname{sgn}\left(\sigma \sigma^{\prime}\right) \mathcal{M}\left(\lambda_{1}, \lambda_{2}, \sigma, \sigma^{\prime}\right)^{*}\right|_{p \leftrightarrow p^{\prime}},
\end{aligned}
$$

where the substitution $p \leftrightarrow p^{\prime}$ also includes the interchange of the respective spinors $\kappa_{i}$ and $\kappa_{i}^{\prime}$.

\section{B. Process $\overline{f f} \rightarrow \gamma \gamma \gamma$ and related reactions}

Next, we consider fermion-antifermion annihilation into three photons:

$$
f(p, \sigma)+\bar{f}\left(p^{\prime}, \sigma^{\prime}\right) \rightarrow \gamma\left(k_{1}, \lambda_{1}\right)+\gamma\left(k_{2}, \lambda_{2}\right)+\gamma\left(k_{3}, \lambda_{3}\right) .
$$

The momentum matrices $P$ and $P^{\prime}$ for the momenta $p$ and $p^{\prime}$, respectively, are again decomposed into the respective spinors $\kappa_{i, A}$ and $\kappa_{i, A}^{\prime}$, as defined in Eqs. (4.5) and (5.2). The Dirac spinors are generically assigned by

$$
\Psi_{p}^{(+)}=\left(\begin{array}{c}
\phi_{A} \\
\psi^{\dot{A}}
\end{array}\right), \quad \bar{\Psi}_{p^{\prime}}^{(-)}=\left(\psi^{\prime A}, \phi_{\dot{A}}^{\prime}\right)
$$

with the actual insertions

$$
\begin{gathered}
(\phi, \psi)= \begin{cases}\left(\kappa_{1},-\kappa_{2}\right) & \text { for } \sigma=+, \\
\left(\kappa_{2}, \kappa_{1}\right) & \text { for } \sigma=-,\end{cases} \\
\left(\phi^{\prime}, \psi^{\prime}\right)= \begin{cases}\left(\kappa_{1}^{\prime}, \kappa_{2}^{\prime}\right) & \text { for } \sigma^{\prime}=-, \\
\left(-\kappa_{2}^{\prime}, \kappa_{1}^{\prime}\right) & \text { for } \sigma^{\prime}=+.\end{cases}
\end{gathered}
$$

For all helicity configurations, we have $\langle\phi \psi\rangle=\left\langle\psi^{\prime} \phi^{\prime}\right\rangle$ $=m_{f}$. The polarization spinors $\varepsilon_{\lambda_{i}, \dot{A} B}^{*}\left(k_{i}\right)$ for the outgoing photons are defined as in Eqs. (3.20). Following the strategy of Sec. IV C and "crossing" the results (4.11) or (4.12) for the subdiagrams of Fig. 1, the actual calculation of the six diagrams for $f \bar{f} \rightarrow \gamma \gamma \gamma$ (see Fig. 3) is rather simple. In order to minimize the number of generic amplitudes that have to be calculated, we first give the relations that follow from discrete symmetries. Parity, CP, and Bose symmetry imply 


$$
\begin{aligned}
\mathcal{M}\left(-\sigma,-\sigma^{\prime},-\lambda_{1},-\lambda_{2},-\lambda_{3}\right) & =-\operatorname{sgn}\left(\sigma \sigma^{\prime}\right) \mathcal{M}\left(\sigma, \sigma^{\prime}, \lambda_{1}, \lambda_{2}, \lambda_{3}\right)^{*}, \\
\mathcal{M}\left(-\sigma^{\prime},-\sigma,-\lambda_{1},-\lambda_{2},-\lambda_{3}\right) & =-\left.\operatorname{sgn}\left(\sigma \sigma^{\prime}\right) \mathcal{M}\left(\sigma, \sigma^{\prime}, \lambda_{1}, \lambda_{2}, \lambda_{3}\right)^{*}\right|_{p \leftrightarrow p^{\prime}}, \\
\mathcal{M}\left(\sigma, \sigma^{\prime}, \lambda_{i_{1}}, \lambda_{i_{2}}, \lambda_{i_{3}}\right) & =\left.\mathcal{M}\left(\sigma, \sigma^{\prime}, \lambda_{1}, \lambda_{2}, \lambda_{3}\right)\right|_{k_{1} \rightarrow k_{i_{1}}, k_{2} \rightarrow k_{i_{2}}, k_{3} \rightarrow k_{i_{3}}} .
\end{aligned}
$$

Therefore, for any given $\left(\sigma, \sigma^{\prime}\right)$ combination, it suffices to calculate the helicity amplitudes with $\left(\lambda_{1}, \lambda_{2}, \lambda_{3}\right)=(+++)$, $(++-)$, from which all other amplitudes follow by Eqs. (5.13). These generic results can be expressed in a very compact form

$$
\mathcal{M}\left(\sigma, \sigma^{\prime}, \lambda_{1}, \lambda_{2}, \lambda_{3}\right)=\frac{Q_{f}^{3} e^{3} A_{\lambda_{1} \lambda_{2} \lambda_{3}}\left(\sigma, \sigma^{\prime}\right)}{4 \sqrt{2}\left(p \cdot k_{1}\right)\left(p \cdot k_{2}\right)\left(p \cdot k_{3}\right)},
$$

where

$$
\begin{aligned}
A_{+++}\left(\sigma, \sigma^{\prime}\right)= & m_{f} \frac{\left(\left\langle k_{2} k_{3}\right\rangle^{*}\right)^{2}}{\left(p^{\prime} \cdot k_{1}\right)}\left\langle k_{1} P^{\prime} P k_{1}\right\rangle\left\langle\phi \psi^{\prime}\right\rangle+\text { cyclic permutations in } k_{1}, k_{2}, k_{3}, \\
A_{++-}\left(\sigma, \sigma^{\prime}\right)= & m_{f} \frac{\left(\left\langle k_{1} k_{2}\right\rangle^{*}\right)^{2}}{2\left(p^{\prime} \cdot k_{3}\right)}\left(\left\langle k_{3} P^{\prime} P k_{3}\right\rangle^{*}\left\langle\phi \psi^{\prime}\right\rangle+2\left(p \cdot k_{3}\right)\left\langle k_{3} \phi\right\rangle\left\langle k_{3} \psi^{\prime}\right\rangle\right) \\
& -\frac{\left\langle k_{2} P k_{3}\right\rangle}{\left(p^{\prime} \cdot k_{1}\right)}\left[\left\langle k_{1} P^{\prime} P k_{1}\right\rangle\left(\left\langle k_{2} \psi\right\rangle^{*}\left\langle k_{3} \psi^{\prime}\right\rangle+\left\langle k_{2} \phi^{\prime}\right\rangle *\left\langle k_{3} \phi\right\rangle\right)+2\left(p \cdot k_{1}\right)\left\langle k_{3} \phi\right\rangle\left\langle k_{1} \phi^{\prime}\right\rangle^{*}\left\langle k_{1} k_{2}\right\rangle^{*}\right]+\left(k_{1} \leftrightarrow k_{2}\right) .
\end{aligned}
$$

In addition to the shorthand $\left\langle k_{l} P k_{n}\right\rangle$ of Eq. (5.7) we have introduced the useful abbreviations

$$
\begin{gathered}
\left\langle k_{l} P^{\prime} P k_{l}\right\rangle=k_{l, \dot{A}} P^{\prime \dot{A} B} P_{\dot{C} B} k_{l}^{\dot{C}}=\sum_{i, j=1,2}\left\langle k_{l} \kappa_{i}^{\prime}\right\rangle *\left\langle\kappa_{j} \kappa_{i}^{\prime}\right\rangle\left\langle\kappa_{j} k_{l}\right\rangle^{*}, \\
l=1,2,3 .
\end{gathered}
$$

The above results for $f \bar{f} \rightarrow \gamma \gamma \gamma$ can also be used to generate the helicity amplitudes for the bremsstrahlung processes $\gamma \gamma \rightarrow f \bar{f} \gamma$ and $e^{-} \gamma \rightarrow e^{-} \gamma \gamma$, if the crossing relations of Sec. IV B are applied. In particular, we get a set of helicity amplitudes for the so-called double Compton process

$$
e^{-}(p, \sigma)+\gamma(k, \lambda) \rightarrow e^{-}\left(p^{\prime}, \sigma^{\prime}\right)+\gamma\left(k_{1}^{\prime}, \lambda_{1}^{\prime}\right)+\gamma\left(k_{2}^{\prime}, \lambda_{2}^{\prime}\right)
$$

by identifying $f=e^{-}$, consistently replacing

$$
\begin{gathered}
k_{3}^{\mu} \rightarrow-k^{\mu}, \quad k_{3, A} \rightarrow k_{3, A}, \quad k_{3, \dot{A}} \rightarrow-k_{3, \dot{A}}, \quad \lambda_{3} \rightarrow-\lambda, \\
p^{\prime \mu} \rightarrow-p^{\prime \mu}, \quad \kappa_{i, A}^{\prime} \rightarrow \kappa_{i, A}^{\prime}, \quad \kappa_{i, \dot{A}}^{\prime} \rightarrow-\kappa_{i, \dot{A}}^{\prime}, \quad \sigma^{\prime} \rightarrow-\sigma^{\prime}
\end{gathered}
$$

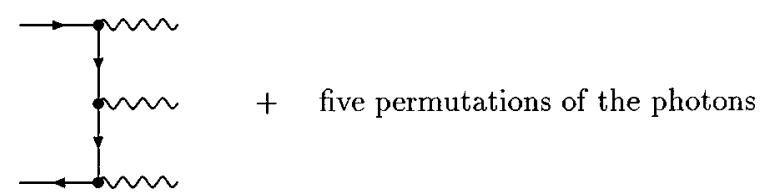

FIG. 3. Born diagrams for $f \bar{f} \rightarrow \gamma \gamma \gamma$. in all expressions, and adding the factor $\operatorname{sgn}\left(\sigma^{\prime}\right)$, where $\sigma^{\prime}$ is the helicity of the outgoing $e^{-}$in Eq. (5.17). Finally, we mention that our helicity amplitudes have been numerically reproduced (up to phase factors) in the framework of the calculation presented in Ref. [14] by employing the spinor method of Ref. [2] and by a third, completely different method.

\section{Process $\mu^{-} \mu^{+} \rightarrow f \bar{f} \gamma$ and related reactions}

As a final example, we consider the process

$$
\mu^{-}(p, \sigma)+\mu^{+}\left(p^{\prime}, \sigma^{\prime}\right) \rightarrow f(q, \tau)+\bar{f}\left(q^{\prime}, \tau^{\prime}\right)+\gamma(k, \lambda),
$$

which will be of relevance at future muon colliders. The momenta $p, p^{\prime}$ and the corresponding Dirac spinors $\Psi_{p}^{(+)}, \bar{\Psi}_{p^{\prime}}^{(-)}$for the incoming muons are defined in the same way as in the last section [see Eqs. (5.11) and (5.12)]. The momentum matrices $Q$ and $Q^{\prime}$ for the outgoing momenta $q$ and $q^{\prime}$ are decomposed into the spinors $\rho_{i, A}$ and $\rho_{i, A}^{\prime}$, respectively:

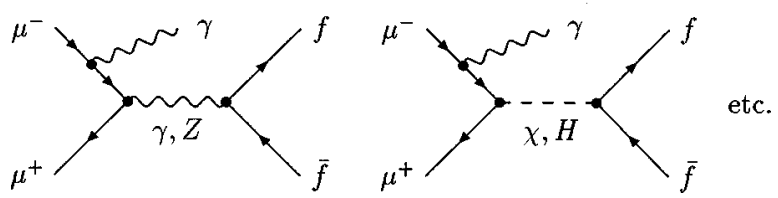

FIG. 4. Born diagrams for $\mu^{-} \mu^{+} \rightarrow f \bar{f} \gamma$, where graphs with the outgoing photon attached to the other charged fields are suppressed. 


$$
Q_{\dot{A} B}=\sum_{i=1,2} \rho_{i, \dot{A}} \rho_{i, B}, \quad Q_{\dot{A} B}^{\prime}=\sum_{i=1,2} \rho_{i, \dot{A}}^{\prime} \rho_{i, B}^{\prime}
$$

The respective Dirac spinors are generically given by

$$
\bar{\Psi}_{q}^{(+)}=\left(\eta^{A}, \xi_{\dot{A}}\right), \quad \Psi_{q^{\prime}}^{(-)}=\left(\begin{array}{c}
\xi_{A}^{\prime} \\
\eta^{\prime \dot{A}}
\end{array}\right),
$$

with the actual insertions

$$
\begin{aligned}
& (\xi, \eta)= \begin{cases}\left(\rho_{1},-\rho_{2}\right) & \text { for } \tau=+, \\
\left(\rho_{2}, \rho_{1}\right) & \text { for } \tau=-,\end{cases} \\
& \left(\xi^{\prime}, \eta^{\prime}\right)= \begin{cases}\left(\rho_{1}^{\prime}, \rho_{2}^{\prime}\right) & \text { for } \tau^{\prime}=-, \\
\left(-\rho_{2}^{\prime}, \rho_{1}^{\prime}\right) & \text { for } \tau^{\prime}=+;\end{cases}
\end{aligned}
$$

i.e., we have $\langle\xi \eta\rangle=\left\langle\eta^{\prime} \xi^{\prime}\right\rangle=m_{f}$. The polarization spinors $\varepsilon_{\lambda, A B}^{*}(k)$ for the outgoing photon are defined as in Eqs. (3.20). For $f \neq \mu^{-}, 16$ diagrams contribute to the process in the standard model at the tree level; they are schematically indicated in Fig. 4. The case $f=\mu^{-}$will not be considered here explicitly; the results for this particular channel follow from the ones for $f \neq \mu^{-}$by adding the amplitudes for the crossed process $f \mu^{+} \rightarrow f \mu^{+} \gamma$ with a negative sign. Since the interaction is mediated by the exchange of neutral particles only, the electromagnetic currents of the muon and fermion $f$ are conserved separately. This means that the gauge spinor for the outgoing photon can be chosen differently for initialand final-state radiation, which simplifies the calculation. In the following we generically denote the couplings of the neutral bosons to the fermions by

$$
\begin{aligned}
& g_{\gamma f}^{ \pm}=-Q_{f}, \quad g_{Z f}^{+}=-\frac{s_{w}}{c_{w}} Q_{f}, \quad g_{Z f}^{-}=-\frac{s_{w}}{c_{w}} Q_{f}+\frac{I_{W, f}}{c_{w} s_{w}}, \\
& g_{H f}^{ \pm}=-\frac{1}{2 s_{w}} \frac{m_{f}}{M_{W}}, \quad g_{\chi f}^{ \pm}= \pm \frac{i I_{W, f}}{s_{w}} \frac{m_{f}}{M_{W}}
\end{aligned}
$$

where $I_{W, f}= \pm \frac{1}{2}$ is the weak isospin of the left-handed part of the fermion field $f$. In Eqs. (5.23) we follow the conventions of Ref. [12] for the standard model parameters and fields; in particular, $\chi$ denotes the would-be Goldstone partner to the $Z$ boson, and $H$ is the physical Higgs boson. Since standard electroweak theory does not conserve parity, the parity-induced relations between helicity amplitudes with opposite helicity configurations involve also an interchange of the chiral couplings:

$$
\begin{aligned}
\mathcal{M}( & \left.-\sigma,-\sigma^{\prime},-\tau,-\tau^{\prime},-\lambda\right) \\
& =\left.\operatorname{sgn}\left(\sigma \sigma^{\prime} \tau \tau^{\prime}\right) \mathcal{M}\left(\sigma, \sigma^{\prime}, \tau, \tau^{\prime}, \lambda\right)^{*}\right|_{\left(g_{\ldots}^{ \pm}\right) * \leftrightarrow g_{\ldots}^{\mp} .}
\end{aligned}
$$

On the other hand, the process is $C P$ symmetric at the considered perturbative level, and the relations induced by $C P$ symmetry read

$$
\begin{aligned}
\mathcal{M}( & \left.-\sigma^{\prime},-\sigma,-\tau^{\prime},-\tau,-\lambda\right) \\
& =-\left.\operatorname{sgn}\left(\sigma \sigma^{\prime} \tau \tau^{\prime}\right) \mathcal{M}\left(\sigma, \sigma^{\prime}, \tau, \tau^{\prime}, \lambda\right)^{*}\right|_{p \leftrightarrow p^{\prime}, q \leftrightarrow q^{\prime}} .
\end{aligned}
$$

The matrix elements $\mathcal{M}$ are decomposed according to the boson in the $s$ channel,

$$
\begin{aligned}
\mathcal{M}\left(\sigma, \sigma^{\prime}, \tau, \tau^{\prime}, \lambda\right)= & \sqrt{2} e^{3}\left[\sum_{V=\gamma, Z} A_{\lambda}^{(V)}\left(\sigma, \sigma^{\prime}, \tau, \tau^{\prime}\right)\right. \\
& \left.+\sum_{S=\chi, H} A_{\lambda}^{(S)}\left(\sigma, \sigma^{\prime}, \tau, \tau^{\prime}\right)\right],
\end{aligned}
$$

leading to two generic functions $A_{\lambda}^{(V)}$ and $A_{\lambda}^{(S)}$ for the exchange of a vector field and a scalar field, respectively. Owing to Eqs. (5.24) or (5.25), it is sufficient to give the results for $\lambda=+$ :

$$
\begin{aligned}
A_{+}^{(V)}\left(\sigma, \sigma^{\prime}, \tau, \tau^{\prime}\right)= & \left\{\frac{Q_{\mu}\left\langle k P^{\prime} P k\right\rangle}{2(p \cdot k)\left(p^{\prime} \cdot k\right)\left[\left(q+q^{\prime}\right)^{2}-M_{V}^{2}\right]}-\frac{Q_{f}\left\langle k Q^{\prime} Q k\right\rangle}{2(q \cdot k)\left(q^{\prime} \cdot k\right)\left[\left(p+p^{\prime}\right)^{2}-M_{V}^{2}\right]}\right\} \\
& \times\left(g_{V \mu}^{+} g_{V f}^{+}\left\langle\phi^{\prime} \xi\right\rangle^{*}\left\langle\phi \xi^{\prime}\right\rangle+g_{V \mu}^{+} g_{V f}^{-}\left\langle\phi^{\prime} \eta^{\prime}\right\rangle^{*}\langle\phi \eta\rangle+g_{V \mu}^{-} g_{V f}^{+}\langle\psi \xi\rangle^{*}\left\langle\psi^{\prime} \xi^{\prime}\right\rangle+g_{V \mu}^{-} g_{V f}^{-}\left\langle\psi \eta^{\prime}\right\rangle *\left\langle\psi^{\prime} \eta\right\rangle\right) \\
& -\frac{Q_{\mu}}{\left(q+q^{\prime}\right)^{2}-M_{V}^{2}}\left[\frac{g_{V \mu}^{+}\left\langle k \phi^{\prime}\right\rangle^{*}}{\left(p^{\prime} \cdot k\right)}\left(g_{V f}^{+}\langle k \xi\rangle^{*}\left\langle\phi \xi^{\prime}\right\rangle+g_{V f}^{-}\left\langle k \eta^{\prime}\right\rangle^{*}\langle\phi \eta\rangle\right)\right. \\
& \left.-\frac{g_{V \mu}^{-}\langle k \psi\rangle^{*}}{(p \cdot k)}\left(g_{V f}^{+}\langle k \xi\rangle^{*}\left\langle\psi^{\prime} \xi^{\prime}\right\rangle+g_{V f}^{-}\left\langle k \eta^{\prime}\right\rangle^{*}\left\langle\psi^{\prime} \eta\right\rangle\right)\right] \\
& -\frac{Q_{f}}{\left(p+p^{\prime}\right)^{2}-M_{V}^{2}}\left[\frac{g_{V f}^{+}\langle k \xi\rangle^{*}}{(q \cdot k)}\left(g_{V \mu}^{+}\left\langle k \phi^{\prime}\right\rangle^{*}\left\langle\phi \xi^{\prime}\right\rangle+g_{V \mu}^{-}\langle k \psi\rangle^{*}\left\langle\psi^{\prime} \xi^{\prime}\right\rangle\right)\right. \\
& \left.-\frac{g_{V f}^{-}\left\langle k \eta^{\prime}\right\rangle^{*}}{\left(q^{\prime} \cdot k\right)}\left(g_{V \mu}^{+}\left\langle k \phi^{\prime}\right\rangle *\langle\phi \eta\rangle+g_{V \mu}^{-}\langle k \psi\rangle^{*}\left\langle\psi^{\prime} \eta\right\rangle\right)\right],
\end{aligned}
$$




$$
\begin{aligned}
A_{+}^{(S)}\left(\sigma, \sigma^{\prime}, \tau, \tau^{\prime}\right)= & \left\{\frac{Q_{f}\left\langle k Q^{\prime} Q k\right\rangle}{4(q \cdot k)\left(q^{\prime} \cdot k\right)\left[\left(p+p^{\prime}\right)^{2}-M_{S}^{2}\right]}-\frac{Q_{\mu}\left\langle k P^{\prime} P k\right\rangle}{4(p \cdot k)\left(p^{\prime} \cdot k\right)\left[\left(q+q^{\prime}\right)^{2}-M_{S}^{2}\right]}\right\} \\
& \times\left(g_{S \mu}^{+}\left\langle\phi \psi^{\prime}\right\rangle+g_{S \mu}^{-}\left\langle\phi^{\prime} \psi\right\rangle^{*}\right)\left(g_{S f}^{+}\left\langle\xi^{\prime} \eta\right\rangle+g_{S f}^{-}\left\langle\xi \eta^{\prime}\right\rangle^{*}\right) \\
& +\frac{Q_{\mu} g_{S \mu}^{-}\left\langle k \phi^{\prime}\right\rangle^{*}\langle k \psi\rangle^{*}}{\left(q+q^{\prime}\right)^{2}-M_{S}^{2}}\left(g_{S f}^{+}\left\langle\xi^{\prime} \eta\right\rangle+g_{S f}^{-}\left\langle\xi \eta^{\prime}\right\rangle^{*}\right)\left[\frac{1}{2(p \cdot k)}+\frac{1}{2\left(p^{\prime} \cdot k\right)}\right] \\
& -\frac{Q_{f} g_{S f}^{-}\langle k \xi\rangle^{*}\left\langle k \eta^{\prime}\right\rangle^{*}}{\left(p+p^{\prime}\right)^{2}-M_{S}^{2}}\left(g_{S \mu}^{+}\left\langle\phi \psi^{\prime}\right\rangle+g_{S \mu}^{-}\left\langle\phi^{\prime} \psi\right\rangle^{*}\right)\left[\frac{1}{2(q \cdot k)}+\frac{1}{2\left(q^{\prime} \cdot k\right)}\right] .
\end{aligned}
$$

The quantities $\left\langle k P^{\prime} P k\right\rangle$ and $\left\langle k Q^{\prime} Q k\right\rangle$ are defined in complete analogy to $\left\langle k_{l} P^{\prime} P k_{l}\right\rangle$ in Eq. (5.16). The results for $A_{\lambda}^{(V)}$ and $A_{\lambda}^{(S)}$ are written down in the 't Hooft-Feynman gauge; i.e., we have $M_{\chi}=M_{Z}$. We mention, however, that we have reproduced the result for the complete matrix element $\mathcal{M}$ also in an arbitrary $R_{\xi}$ gauge for the photon and Z-boson fields, in which the individual contributions $A_{\lambda}^{(V)}$ and $A_{\lambda}^{(S)}$ look different.

The above results have also been used to derive the helicity amplitudes for the process

$$
e^{-}(p, \sigma)+\gamma(k, \lambda) \rightarrow e^{-}\left(p^{\prime}, \sigma^{\prime}\right)+e^{-}(q, \tau)+e^{+}\left(q^{\prime}, \tau^{\prime}\right)
$$

in QED. The QED part of the amplitudes (5.26) is obtained by taking the contribution of $V=\gamma$ in Eq. (5.26) only. Moreover, we identify $Q_{\mu}=Q_{f}=Q_{e}=-1$. The crossing proceeds analogously to the case described at the end of the previous section; i.e., one has to perform the replacements

$$
\begin{gathered}
k^{\mu} \rightarrow-k^{\mu}, \quad k_{A} \rightarrow k_{A}, \quad k_{\dot{A}} \rightarrow-k_{\dot{A}}, \quad \lambda \rightarrow-\lambda, \\
p^{\prime \mu} \rightarrow-p^{\prime \mu}, \quad \kappa_{i, A}^{\prime} \rightarrow \kappa_{i, A}^{\prime}, \quad \kappa_{i, \dot{A}}^{\prime} \rightarrow-\kappa_{i, \dot{A}}^{\prime}, \quad \sigma^{\prime} \rightarrow-\sigma^{\prime},
\end{gathered}
$$

and to apply the sign factor $\operatorname{sgn}\left(\sigma^{\prime}\right)$, where $\sigma^{\prime}$ is the helicity of the outgoing $e^{-}$in Eq. (5.28). Finally, we have to antisymmetrize all amplitudes with respect to the interchange of the two outgoing electrons. Moreover, the amplitudes have been numerically reproduced by an independent helicity method in the framework of the calculation discussed in Ref. [14]. The polarized cross sections of $e^{-} \gamma \rightarrow e^{-} e^{-} e^{+}$contribute, in particular, to the left-right asymmetry $A_{L R}$ of polarized Compton scattering as background. The numerical agreement of the results presented in Ref. [14] for this contribution to $A_{L R}$ with the completely independent ones of Ref. [15] represents an additional check of the calculation.

\section{SUMMARY}

The Weyl-van der Waerden (WvdW) spinor technique for the calculation of helicity amplitudes of massive and massless particles has been described in detail, providing all necessary ingredients for an implementation in computer algebra. This formalism leads to rather compact results for amplitudes, which can be directly used for numerical evalu- ations, immediately after all spinor indices have been contracted to so-called spinor products. These contractions are technically similar to the usual ones for Lorentz indices. Since all mathematical objects, such as momenta, polarization vectors, and Dirac spinors, are expressed in terms of WvdW spinors, the spinor calculus often allows for further simplifications at the analytical level. Moreover, we have formulated how to exploit discrete symmetries for a reduction of the algebraic work, or for providing additional checks, and how to avoid problems that are due to the appearance of unphysical poles in amplitudes.

The use and the power of the described spinor technique have been demonstrated by the explicit calculation of the helicity amplitudes for the processes $\gamma \gamma \rightarrow f \bar{f}, f \bar{f} \rightarrow \gamma \gamma \gamma$, $\mu^{-} \mu^{+} \rightarrow f \bar{f} \gamma$ with massive fermions and of reactions that can be obtained from those by crossing symmetry. The results, which have been analytically simplified as far as possible, are very compact and well suited to numerical evaluations.

\section{ACKNOWLEDGMENTS}

The author thanks D. de Florian, A. Denner, and W. Vogelsang for a critical reading of the manuscript and for their aid in making the presentation as transparent as possible.

\section{APPENDIX: MORE DETAILS ABOUT DISCRETE SYMMETRIES}

In this appendix we supplement the discussion of parity and $C P$ symmetry in Sec. IV B by further details.

\section{Parity}

In order to explicitly derive the relations between helicity amplitudes that are connected by parity, we inspect the individual terms in an amplitude $\mathcal{M}$ after complex conjugation. Contractions between momentum matrices, which are just Minkowski inner products according to Eq. (2.13), are not changed at all, since the result is real. Contractions between a momentum $k$ and a polarization vector $\varepsilon$ or between two polarization vectors turn into the products for the corresponding polarization vectors with opposite helicity, since $\left(K_{\dot{A} B}\right)^{*}=K_{A \dot{B}}=K_{\dot{B} A}$ and $\left(\varepsilon_{\lambda, \dot{A} B}\right)^{*}=\varepsilon_{\lambda, A \dot{B}}=\varepsilon_{-\lambda, \dot{B} A}(\lambda=0$, $\pm 1)$. Coupling factors $i C$ for interactions between bosons 
simply turn into $-i C^{*}$, and boson propagators receive a factor -1 owing to the explicit factor $i$ in the numerator. The only nontrivial terms are those originating from Dirac matrices and fermionic wave functions. The complex conjugate amplitude remains unchanged if we multiply each fermionic vertex by a factor $\Gamma_{P}^{2}=-\mathbf{1}$ from the left and from the right, where the matrix $\Gamma_{P}$ is defined as

$$
\Gamma_{P}=i \gamma^{2} \gamma^{5}=\left(\begin{array}{ll}
0 & \epsilon \\
\epsilon & 0
\end{array}\right)
$$

Shifting then a factor $\Gamma_{P}$ to the fermion propagators or fermionic wave functions that are attached on the right side of this vertex and shifting a factor $-\Gamma_{P}$ to the left side, we get the following replacements when going over from $\mathcal{M}$ to $\mathcal{M}^{*}$ :

$$
\begin{aligned}
& \text { ie } k C_{V \bar{f}_{1} f_{2}}^{\tau} \omega_{\tau} \rightarrow-\Gamma_{P}\left[\text { ieht } C_{V \bar{f}_{1} f_{2}}^{\tau} \omega_{\tau}\right]^{*} \Gamma_{P} \\
& =-i e \not k\left(C_{V \bar{f}_{1} f_{2}}^{-\tau}\right) * \omega_{\tau}, \\
& i e \oiint_{\lambda} C_{V \bar{f}_{1} f_{2}}^{\tau} \omega_{\tau} \rightarrow-\Gamma_{P}\left[i e d_{\lambda} C_{V \bar{f}_{1} f_{2}}^{\tau} \omega_{\tau}\right]^{*} \Gamma_{P} \\
& =-i e \oiint_{-\lambda}\left(C_{V \bar{f}_{1} f_{2}}^{-\tau}\right) * \omega_{\tau}, \\
& i e C_{S \bar{f}_{1} f_{2}}^{\tau} \omega_{\tau} \rightarrow-\Gamma_{P}\left[i e C_{S \bar{f}_{1} f_{2}}^{\tau} \omega_{\tau}\right] * \Gamma_{P}=-i e\left(C_{S \bar{f}_{1} f_{2}}^{-\tau}\right)^{*} \omega_{\tau}, \\
& i\left(\not h+m_{f}\right) \rightarrow \Gamma_{P}\left[i\left(\not h+m_{f}\right)\right]^{*}\left(-\Gamma_{P}\right)=-i\left(\not h+m_{f}\right), \\
& \Psi_{k, 1}^{( \pm)} \rightarrow \Gamma_{P}\left[\Psi_{k, 1}^{( \pm)}\right]^{*}=+\Psi_{k, 2}^{( \pm)} \\
& \bar{\Psi}_{k, 1}^{( \pm)} \rightarrow\left[\bar{\Psi}_{k, 1}^{( \pm)}\right]^{*}\left(-\Gamma_{P}\right)=+\bar{\Psi}_{k, 2}^{( \pm)}, \\
& \Psi_{k, 2}^{( \pm)} \rightarrow \Gamma_{P}\left[\Psi_{k, 2}^{( \pm)}\right]^{*}=-\Psi_{k, 1}^{( \pm)} \\
& \bar{\Psi}_{k, 2}^{( \pm)} \rightarrow\left[\bar{\Psi}_{k, 2}^{( \pm)}\right]^{*}\left(-\Gamma_{P}\right)=-\bar{\Psi}_{k, 1}^{( \pm)},
\end{aligned}
$$

where the notation for the generic Feynman rules of Tables I and II is used. The relations between $\mathcal{M}\left(\sigma_{i} ; \lambda_{j}\right) *$ and $\mathcal{M}\left(-\sigma_{i} ;-\lambda_{j}\right)$ can be read off from Eqs. (A2) and the above considerations. The substitutions for the chiral couplings are explicitly given; in particular, there is no substitution in parity-conserving theories, in which $C_{V \bar{f}_{1} f_{2}}^{\tau}=\left(C_{V \bar{f}_{1} f_{2}}^{-\tau}\right)^{*}$, etc. There is a global change of sign due to vertices and propagators, since each of them introduces a factor -1 and since their total number is always odd in tree-level amplitudes. Another factor -1 comes from our convention of extracting the factor $i$ from the amplitude $i \mathcal{M}$. Finally, we encounter a factor $\operatorname{sgn}\left(\sigma_{i}\right)$ for each external fermion with helicity $\sigma_{i}$ and a factor $-\operatorname{sgn}\left(\sigma_{i}\right)$ for each external antifermion, leading us directly to relation (4.3).

\section{2. $C P$ symmetry}

As in the case of parity, we derive the $C P$-induced relations between helicity amplitudes by considering the complex conjugate of a given amplitude $\mathcal{M}$. We assume an un- derlying model in which all couplings between bosons are $C P$-conserving, as is the case in the electroweak standard model. Then, for bosonic fields, the situation is simple, because the wave functions of particles and antiparticles are formally identical, and the bosonic couplings that are related by charge conjugation differ by at most the sign factors (see, e.g., the Feynman rules in Ref. [12]). Specifically, there is a factor $(-1)^{v}$ between each vertex factor $i C$ and the expression $i \bar{C}$ for the vertex involving the corresponding chargeconjugated fields, where $v$ is the number of vector bosons attached to the vertex. In order to get rid of the factor $(-1)^{v}$, we shift a factor -1 for each vector-boson leg of a vertex to the attached vector-boson propagators or external wave functions, resulting in an overall factor $(-1)^{n_{V}}$ for each diagram, where $n_{V}$ is the number of external vector bosons. After this procedure the bosonic propagators and vertices for the charge-conjugated fields consistently differ by a factor -1 from the complex-conjugated counterparts involving the original fields.

For fermionic fields we proceed in a way similar to the treatment of parity described above. In addition to taking the complex conjugate of a Dirac chain, we now also transpose each term and invert the order in the chain, since charge conjugation reverses the directions of fermionic lines. Then we multiply fermionic vertices, propagators, and wave functions by the matrix

$$
\Gamma_{C P}=-\gamma^{0} \gamma^{5}=\left(\begin{array}{cc}
0 & \mathbf{1} \\
-\mathbf{1} & 0
\end{array}\right)
$$

with $\Gamma_{C P}^{2}=-\mathbf{1}$ in the same way as done with $\Gamma_{P}$ above. This leads to the replacements

$$
\begin{aligned}
& \text { iekk } C_{V \bar{f}_{1} f_{2}}^{\tau} \omega_{\tau} \rightarrow-\Gamma_{C P}\left[-i e \not k C_{V \bar{f}_{1} f_{2}}^{\tau} \omega_{\tau}\right]^{\dagger} \Gamma_{C P} \\
& =-i e \not k\left(C_{V \bar{f}_{1} f_{2}}^{\tau}\right) * \omega_{\tau}, \\
& i e d_{\lambda} C_{V \bar{f}_{1} f_{2}}^{\tau} \omega_{\tau} \rightarrow-\Gamma_{C P}\left[-i e d_{\lambda} C_{V \bar{f}_{1} f_{2}}^{\tau} \omega_{\tau}\right]^{\dagger} \Gamma_{C P} \\
& =-i e d_{-\lambda}\left(C_{V \bar{f}_{1} f_{2}}^{\tau}\right) * \omega_{\tau}, \\
& i e C_{S \bar{f}_{1} f_{2}}^{\tau} \omega_{\tau} \rightarrow-\Gamma_{C P}\left[i e C_{S \bar{f}_{1} f_{2}}^{\tau} \omega_{\tau}\right]^{\dagger} \Gamma_{C P} \\
& =-i e\left(C_{S \bar{f}_{1} f_{2}}^{-\tau}\right) * \omega_{\tau}, \\
& i\left(\not k+m_{f}\right) \rightarrow \Gamma_{C P}\left[i\left(\not k+m_{f}\right)\right]^{\dagger}\left(-\Gamma_{C P}\right) \\
& =-i\left(-\not h+m_{f}\right) \text {, } \\
& \Psi_{k, 1}^{( \pm)} \rightarrow\left[\Psi_{k, 1}^{( \pm)}\right]^{\dagger}\left(-\Gamma_{C P}\right)=-\Psi_{k, 1}^{(\mp)}, \\
& \bar{\Psi}_{k, 1}^{( \pm)} \rightarrow \Gamma_{C P}\left[\bar{\Psi}_{k, 1}^{( \pm)}\right]^{\dagger}=+\Psi_{k, 1}^{(\mp)} \\
& \Psi_{k, 2}^{( \pm)} \rightarrow\left[\Psi_{k, 2}^{( \pm)}\right]^{\dagger}\left(-\Gamma_{C P}\right)=+\bar{\Psi}_{k, 2}^{(\mp)}, \\
& \Psi_{k, 2}^{( \pm)} \rightarrow \Gamma_{C P}\left[\Psi_{k, 2}^{( \pm)}\right]^{\dagger}=-\Psi_{k, 2}^{(\mp)} .
\end{aligned}
$$


Note that we had to include a factor -1 for the vectorboson-fermion coupling in the first two substitutions, so as to be consistent with the above treatment of bosonic couplings. Moreover, one should realize the correct change of sign in the momentum of the fermion propagator [see fourth substitution in Eqs. (A4)], corresponding to the inversion of the fermion line. In the case of $C P$ violation, the necessary substitutions for the couplings can be read off from Eqs. (A4); if $C P$ is conserved, no substitution is necessary, because then $C_{V \bar{f}_{1} f_{2}}^{\tau}=\left(C_{V \bar{f}_{1} f_{2}}^{\tau}\right)^{*}$ and $C_{S \bar{f}_{1} f_{2}}^{\tau}=\left(C_{S \bar{f}_{1} f_{2}}^{-\tau}\right)^{*}$. The overall sign between the amplitude $\mathcal{M}\left(\sigma_{i} ; \lambda_{j}\right) *$ and the one for the $C P$-related process $\overline{\mathcal{M}}\left(-\sigma_{i} ;-\lambda_{j}\right)$ is deduced as follows. At the tree level, there is an overall factor -1 from the vertices and propagators and another -1 from the convention for $\mathcal{M}$, as in the case of parity. From Eqs. (A4) one can see that incoming fermions and antifermions with helicity $\sigma_{i}$ introduce each a factor $-\operatorname{sgn}\left(\sigma_{i}\right)$, while outgoing fermionic lines yield a factor $\operatorname{sgn}\left(\sigma_{i}\right)$. Taking into account the factor $(-1)^{n_{V}}$ derived above, we obtain relation (4.4).
[1] P. De Causmaecker et al., Phys. Lett. 105B, 215 (1981); Nucl. Phys. B206, 53 (1982); F. A. Berends et al., ibid. B206, 61 (1982); R. Kleiss, ibid. B241, 61 (1984); J. F. Gunion and Z. Kunszt, Phys. Lett. 159B, 167 (1985); 161B, 333 (1985).

[2] G. Passarino, Nucl. Phys. B237, 249 (1984); F. A. Berends, P. H. Daverveldt, and R. Kleiss, ibid. B253, 441 (1985); R. Kleiss and W. J. Stirling, ibid. B262, 235 (1985); Phys. Lett. B 179, 159 (1986); R. Kleiss, Z. Phys. C 33, 433 (1987).

[3] H. Weyl, The Theory of Groups and Quantum Mechanics (Dover, New York, 1931); B. L. van der Waerden, Group Theory and Quantum Mechanics (Springer-Verlag, Berlin, 1974).

[4] P. A. Carruthers, Spin and Isospin in Particle Physics (Gordon and Breach, New York, 1971).

[5] G. R. Farrar and F. Neri, Phys. Lett. 130B, 109 (1983); 152B, 443(E) (1985); F. A. Berends and W. T. Giele, Nucl. Phys. B294, 700 (1987); B306, 759 (1988); B313, 595 (1989); F. A. Berends, W. T. Giele, and H. Kuijf, ibid. B321, 39 (1989); B333, 120 (1990); S. Dittmaier, ibid. B423, 384 (1994).
[6] W. Beenakker, F. A. Berends, and T. Sack, Nucl. Phys. B367, 287 (1991).

[7] M. Böhm and S. Dittmaier, Nucl. Phys. B409, 3 (1993); B412, 39 (1994).

[8] T. B. Anders and W. Jachmann, J. Math. Phys. 24, 2847 (1983).

[9] W. T. Giele, Ph.D. thesis, University of Leiden, 1989; H. Kuijf, Ph.D. thesis, University of Leiden, 1991.

[10] S. Dittmaier, Ph.D. thesis, University of Würzburg, 1993.

[11] E. Accomando et al., Phys. Rep. 299, 1 (1998).

[12] M. Böhm, W. Hollik, and H. Spiesberger, Fortschr. Phys. 34, 687 (1986); A. Denner, ibid. 41, 307 (1993); A. Denner, S. Dittmaier, and G. Weiglein, Nucl. Phys. B440, 95 (1995).

[13] S. Wolfram, Mathematica- A System for Doing Mathematics by Computer (Addison-Wesley, Redwood City, CA, 1988).

[14] A. Denner and S. Dittmaier, Report No. CERN-TH/98-142, hep-ph/9805443.

[15] M. L. Swartz, Phys. Rev. D 58, 014010 (1998). 